\title{
EB lifetime distributions as alternative to the EP lifetime distributions
}

\author{
Carmen Elena Lupu, Sergiu Lupu, Adina Petcu
}

\begin{abstract}
In this paper we consider lifetime distributions called EB-Max distribution and EB-Min. In the conditions of the Poisson's Limit Theorem it is shown that EB-Max distribution may be approximated by its analogous called EP-Max lifetime distribution and EB-Min distribution may be approximated by its analogous EP-Min lifetime distribution. Further, as example, two methods are provided to simulate pseudo random number for EB-Min distribution and we apply EM algorithm to estimate parameters of EB-Min distribution. An example with real data is also presented and the proposed simulation algorithms where implemented in Maple.
\end{abstract}

\section{Introduction}

In paper [6] it was introduced EB-Min distribution compounding exponentially distributed lifetimes with zero truncated binomially distributed r.v. as alternative to the EP-Min lifetime distribution introduced by Kuş, C. [5] mixing the same lifetime with zero truncated Poisson distributed r.v.. In the both cases lifetimes are represented as minimum of $k$ exponentially distributed r.v., $k=1,2, \ldots$. If we substitute minimum by maximum we abtain EB-Max $[4,7]$ and EP-max [3] distributions which are a special case of complementary exponential power series (CEPS) distributions introduced in 2012 by Jose Flores D. et al. The purpose of this paper is to study possible connections between $\mathrm{EP}$ and EP lifetime distributions.

Key Words: lifetime distribution, mixing r.v., exponential, zero truncated binomial and Poisson distributions, Poisson's Limit Theorem, statistical simulation, EM algorithm.

2010 Mathematics Subject Classification: Primary 60E05, 60F05.

Received: 4 April, 2013.

Revised: 26 June, 2013

Accepted: 27 June, 2013. 


\section{$2 \quad$ EP and EB lifetime distributions}

First of all, we write a general formula [4] for distribution of r.v. $\max \left(W_{1}\right.$, $\left.W_{2}, \ldots, W_{K}\right)$, where $\left(W_{i}\right)_{i \geq 1}$ are independent identically distributed random variables (i.i.d.r.v.) and $K$ is a discrete r.v. such that $\mathbf{P}(K \in\{1,2, \ldots\})=1$. So, we consider that distribution function (d.f.) of r.v. $W_{i}$ is $F(x)=\mathbf{P}\left(W_{i} \leq\right.$ $x), i \geq 1$. Then, due of independence of r.v. $\left(W_{i}\right)_{i \geq 1}$, the d.f. of r.v. $Y_{k}=$ $\max \left(W_{1}, W_{2}, \ldots, W_{k}\right)$ is

$$
F_{Y_{k}}(x)=\mathbf{P}\left(Y_{k} \leq x\right)=[F(x)]^{k}, \forall k=1,2, \ldots .
$$

This means that d.f. of r.v. $Y=\max \left(W_{1}, W_{2}, \ldots, W_{K}\right)$ is a mixture of d.f. $F_{Y_{k}}(x)$ with respect to the distribution of r.v. $K$. Indeed

$$
F_{Y}(x)=\mathbf{P}(Y \leq x)=\mathbf{P}\left(\max \left(W_{1}, W_{2}, \ldots, W_{K}\right) \leq x\right)=\sum_{k \geq 1}[F(x)]^{k} \mathbf{P}(K=k) .(\mathbf{1})
$$

This formula show us that, if $\left(W_{i}\right)_{i \geq 1}$ are r.v. of absolutely continuos type, then $Y$ is a r.v. of the same type and its probability density function (p.d.f.) is

$$
f_{Y}(x)=F_{Y}^{\prime}(x)=\sum_{k \geq 1} k F^{\prime}(x)[F(x)]^{k-1} \mathbf{P}(K=k) \cdot(\mathbf{2})
$$

Similarly, can be write the general formula for distribution of r.v. $Z=$ $\min \left(W_{1}, W_{2}, \ldots, W_{K}\right)$. So, density function and probability density function are

$$
\begin{aligned}
F_{Z}(x) & =\mathbf{P}(Z<x)=1-\mathbf{P}\left(\min \left(W_{1}, W_{2}, \ldots, W_{K}\right) \geqslant x\right) \\
& =1-\left(1-\sum_{k \geq 1}[F(x)]\right)^{k} \mathbf{P}(K=k) .(\mathbf{3}) \\
f_{Z}(x) & =F_{Z}^{\prime}(x)=\sum_{k \geq 1} k F^{\prime}(x)[1-F(x)]^{k-1} \mathbf{P}(K=k) .(\mathbf{4})
\end{aligned}
$$

Applying formulas $(\mathbf{1})-(\mathbf{2})$, and considering that $\left(W_{i}\right)_{i \geq 1}$ are independent identically exponentially distributed r.v. with parameter $\lambda, \lambda>0$, i.e.

$$
F(x)=\mathbf{P}\left(W_{i} \leq x\right)=\left(1-e^{-\lambda x}\right) \cdot I_{[0,+\infty)}(x), i \geq 1
$$

and $K$ is a zero truncated binomially distributed r.v., i.e.,

$$
\mathbf{P}(K=k)=\frac{1}{1-(1-p)^{n}} \mathbb{C}_{n}^{k} p^{k}(1-p)^{n-k}, k=\overline{1 . n}, p \in(0,1),
$$


we obtain the distribution called EB-max $[4,7]$ with d.f. of r.v. $Y=$ $\max \left(W_{1}, W_{2}, \ldots, W_{K}\right)$ given by formula

$$
U_{\max }(x)=\frac{\left(1-p e^{-\lambda x}\right)^{n}-(1-p)^{n}}{1-(1-p)^{n}} \cdot I_{[0,+\infty)}(x)
$$

and p.d.f. of r.v. $Y$ is

$$
u_{\max }(x)=\frac{n p \lambda e^{-\lambda x}}{1-(1-p)^{n}}\left(1-p e^{-\lambda x}\right)^{n} \cdot I_{[0,+\infty)}(x),
$$

where

$$
I_{[0,+\infty)}(x)=\left\{\begin{array}{l}
0, \text { if } x<0 \\
1, \text { if } x \geq 0
\end{array}\right.
$$

For the same lifetimes $\left(W_{i}\right)_{i \geq 1}$, substituting zero truncated binomial distribution for r.v. $K$ by zero truncated Poisson distribution with parameter $\mu$, $\mu>0$, i.e.,

$$
\mathbf{P}(K=k)=\frac{1}{1-e^{-\mu}} \frac{\mu^{k}}{k !} e^{-\mu}, k=1,2, \ldots,
$$

in [3] it was introduced another new lifetime distribution called EP-Max distribution given by formula

$$
V_{\max }(x)=\frac{e^{-\mu e^{-\lambda x}}-e^{-\mu}}{1-e^{-\mu}} \cdot I_{[0,+\infty)}(x) .
$$

In similar way it was introduced EB-Min [6] and EP-Min [5] life time distributions. According to [6] EB-Min distribution is given by d.f.

$$
U_{\min }(x)=\left\{1-\frac{1}{1-(1-p)^{n}}\left\{\left[1-p\left(1-e^{-\lambda x}\right)\right]^{n}-(1-p)^{n}\right\}\right\} \cdot I_{[0,+\infty)}(x) .
$$

and according to[5] EP-Min distribution is given by d.f.

$$
V_{\min }(x)=\frac{e^{\mu e^{-\lambda x}}-e^{\mu}}{1-e^{\mu}} \cdot I_{[0,+\infty)}(x) \cdot(\mathbf{9})
$$

\section{Approximating EB distributions by EP distributions}

As we know [2], Poisson's Limit Theorem show us that, in some conditions, binomial distribution may by approximated by Poisson distribution. This fact suggest us that between d.f. $U_{\max }(x)$ and $V_{\max }(x)$ and, on the other hand, between d.f. $U_{\min }(x)$ and $V_{\min }(x)$ does exist the similar connections. Indeed, it is true the following 
Proposition (Poisson's Limit Theorem for EB an EP distributions). In the conditions of the Poisson's Limit Theorem, i.e., if $n \longrightarrow+\infty$ and

$p \longrightarrow 0$ in a such way that $n p \longrightarrow \mu, \mu>0$, then $[4,7]$

$$
\begin{array}{ll}
\lim _{\substack{\longrightarrow+\infty}} U_{\max }(x)=\lim _{\substack{\longrightarrow \\
p \longrightarrow+\infty}} \frac{\left(1-p e^{-\lambda x}\right)^{n}-(1-p)^{n}}{1-(1-p)^{n}} \cdot I_{[0,+\infty)}(x) \\
& =V_{\max }(x), \forall x \in \mathbb{R}
\end{array}
$$

and

$$
\begin{gathered}
\lim _{\substack{x \\
p \longrightarrow+\infty}} U_{\text {min }}(x)= \\
\lim _{\substack{\longrightarrow \rightarrow+\infty}}\left\{1-\frac{1}{1-(1-p)^{n}}\left\{\left[1-p\left(1-e^{-\lambda x}\right)\right]^{n}-(1-p)^{n}\right\}\right\} \cdot I_{[0,+\infty)}(x) \\
=V_{\min }(x), \forall x \in \mathbb{R} .
\end{gathered}
$$

Remark. Let us observe that the second part of this Proposition will be confirmed empirically in the Example 2 of our work (see Table 5).

\section{On the statistical simulation of EB-Min distribution}

We consider, as example, two techniques for statistical simulation for EB-min distribution $[6,7]$.

The first method is based on the fact that distribution of r.v. $X \sim E B-$ $\operatorname{Min}(\lambda ; n, p) \quad$ coincide with distribution of r.v. $\min _{1 \leqslant i \leqslant Z} W_{i}$, where $\left(W_{i}\right)_{i \geqslant 1}$ are i.i.d.r., $W_{i} \sim \operatorname{Exp}(\lambda), \lambda>0$, and r.v. $Z$ is zero truncated binomially distributed with parameters $n, n \in\{2,3, \ldots\}$ and $p, p \in(0,1)$. So, we deduce the following algorihm

Algorithm MixtEB-Min for statistical generation of number $x$ of r.v. $X \sim E B-\operatorname{Min}(\lambda ; n, p)$.

Step 1. Simulate value $z$ of r.v. $Z \sim \operatorname{Binomial}(n, p)$ until $z$ become different of zero;

Step 2. For this $z$ we simulates values $w_{i}, i=1,2, \ldots z$ as a values of $z$ r.v. i.i.d. $\operatorname{Exp}(\lambda)$;

Step 3. Take $x=\min _{1 \leq i \leq z} w_{i}$. 
The second is enveloping procedure, as a particular case of rejection's Method, presented in [8], which conduct us to the

Algorithm EnvEB-Min for statistical generation of number $x$ of r.v. $X \sim E B-\operatorname{Min}(\lambda ; n, p)$.

From (8) we deduce that p.d.f. of r.v. $X \sim E B-\operatorname{Min}(\lambda ; n, p)$ is given by formula

$$
u_{\min }(x)=\frac{n p \lambda e^{-\lambda x}}{1-(1-p)^{n}}\left[1-p\left(1-e^{-\lambda x}\right)\right]^{n-1} \cdot I_{[0,+\infty)}(x) \cdot(\mathbf{1 0})
$$

For $u_{\min }(x)$ we choose as enveloping p.d.f.

$$
h(x)=\lambda e^{-\lambda x} \cdot I_{[0,+\infty)}(x) .
$$

So, for $\forall x \geq 0$,

$$
r(x)=\frac{u_{\min }(x)}{h(x)}=\frac{n p}{1-(1-p)^{n}}\left[1-p\left(1-e^{-\lambda x}\right)\right]^{n-1},
$$

and

$$
r^{\prime}(x)=-\frac{\lambda n p^{2}(n-1) e^{-\lambda x}}{1-(1-p)^{n}}\left[1-p\left(1-e^{-\lambda x}\right)\right]^{n-2},
$$

We observe that $r^{\prime}(x)<0$, which implies $r(x)<r(0)=\alpha=\frac{n p}{1-(1-p)^{n}}$, where $\alpha>1$.

Step 1. Generate value u of r.v. $U \sim U([0,1])$;

Step 2. If $u<10^{-7}$ then go to Step 1 ;

Step 3. Generate value $u_{1}$ of r.v. $U \sim U([0,1])$;

Step 4. If $u_{1}>(1-p(1-u))^{n-1}$ then go to Step 3;

Step.5. Take $x=-\frac{1}{\lambda} \log (u)$.

The probability of acceptance in this case is

$$
p_{a}=\frac{1}{\alpha}=\frac{1-(1-p)^{n}}{n p} .
$$

In the following Table 1 we have, as a results, CPU's time (in seconds) $\theta(n, p, \lambda)$, executing $N=10000$ simulations in the cases of both algorithms: 
EB LIFETIME DISTRIBUTIONS AS ALTERNATIVE TO THE EP LIFETIME DISTRIBUTIONS

Table 1. CPU's time (in seconds) $\theta(n, p, \lambda)$

\begin{tabular}{|c|c|c|}
\hline$(n, p, \lambda)$ & $\begin{array}{c}\theta(n, p, \lambda)(\mathrm{sec}) \text { for } \\
\text { Algorithm MixtEB-Min }\end{array}$ & $\begin{array}{c}\theta(n, p, \lambda)(\mathrm{sec}) \text { for } \\
\text { Algorithm EnvEB-Min }\end{array}$ \\
\hline$(3,0.5,1)$ & 0.330 & 0.235 \\
\hline$(5,0.5,1)$ & 0.335 & 0.362 \\
\hline$(3,0.5,2)$ & 0.352 & 0.268 \\
\hline$(5,0.5,2)$ & 0.386 & 0.390 \\
\hline$(3,0.9,1)$ & 0.441 & 0.413 \\
\hline$(3,0.9,2)$ & 0.592 & 0.438 \\
\hline$(5,0.9,2)$ & 0.607 & 0.480 \\
\hline$(3,0.2,1)$ & 0.623 & 0.206 \\
\hline$(3,0.2,2)$ & 0.652 & 0.210 \\
\hline$(5,0.2,2)$ & 0.599 & 0.234 \\
\hline$(10,0.5,7)$ & 0.556 & 0.507 \\
\hline$(15,0.5,2)$ & 0.607 & 0.818 \\
\hline
\end{tabular}

On the base of the same simulations we may see (Table 2 and Table 3 ) how look mean value $\mathbb{E} X$, variance $\mathbb{D} X$ and corresponding sample mean value $\bar{x}$ and variance $s^{2}$ for r.v. $X \sim E B-\operatorname{Min}(\lambda ; n, p)$ in case of each Algorithm.

Table 2. Algorithm MixEB-Min

\begin{tabular}{|l|r|c|c|}
\hline \multicolumn{2}{|l|}{ Case 1: $n=3, p=0.5, \lambda=1$} & \multicolumn{2}{|c|}{ Case $2: n=5, p=0.5, \lambda=1$} \\
\hline $\mathbb{E} X=0.690$ & $\mathbb{D} X=0.551$ & $\mathbb{E} X=0.476$ & $\mathbb{D} X=0.289$ \\
\hline $\bar{x}=0.676$ & $s^{2}=0.577$ & $\bar{x}=0.467$ & $s^{2}=0.315$ \\
\hline Case $3: n=3, p=0.5, \lambda=2$ & Case $4: n=5, p=0.5, \lambda=2$ \\
\hline $\mathbb{E} X=0.345$ & $\mathbb{D} X=0.137$ & $\mathbb{E} X=0.238$ & $\mathbb{D} X=0.072$ \\
\hline $\bar{x}=0.324$ & $s^{2}=0.145$ & $\bar{x}=0.234$ & $s^{2}=0.078$ \\
\hline
\end{tabular}

Table 3. Algorithm EnvEB-Min

\begin{tabular}{|c|c|c|c|}
\hline \multicolumn{2}{|c|}{ Case 1: $n=3, p=0.5, \lambda=1$} & \multicolumn{2}{|c|}{ Case 2: $n=5, p=0.5, \lambda=1$} \\
\hline $\mathbb{E} X=0.690$ & $\mathbb{D} X=0.551$ & $E X=0.476$ & $\mathbb{D} X=0.289$ \\
\hline $\bar{x}=0.669$ & $s^{2}=0.553$ & $\bar{x}=0.481$ & $s^{2}=0.298$ \\
\hline \multicolumn{2}{|c|}{ Case 3: $n=3, p=0.5, \lambda=2$} & \multicolumn{2}{|c|}{ Case 4: $n=5, p=0.5, \lambda=2$} \\
\hline $\mathbb{E} X=0.345$ & $\mathbb{D} X=0.137$ & $\mathbb{E} X=0.238$ & $\mathbb{D} X=0.072$ \\
\hline $\bar{x}=0.359$ & $s^{2}=0.151$ & $\bar{x}=0.225$ & $s^{2}=0.069$ \\
\hline
\end{tabular}




\section{EM algorithm and its application to the $E B-M i n$ distribution}

If we consider sample of size $m$ from population of r.v. $X$, i.e., $\left(x_{1}, x_{2}, \ldots\right.$, $\left.x_{m}\right) \sim X$, where $X \sim E B-\operatorname{Min}(\lambda ; n, p)$, then, from (10) we deduce that maximum likelihood function $L\left(x_{1}, x_{2}, \ldots, x_{m} ; n, p, \lambda\right)$ is defined by formula

$$
\begin{gathered}
L\left(x_{1}, x_{2}, \ldots, x_{m} ; \lambda, p\right)=\prod_{i=1}^{m} \frac{n p \lambda \exp \left\{-\lambda x_{i}\right\}}{1-(1-p)^{n}}\left[1-p\left(1-\exp \left\{-\lambda x_{i}\right\}\right)\right]^{n-1}= \\
\frac{(n p \lambda)^{m} \exp \left\{-\lambda \sum_{i=1}^{m} x_{i}\right\}}{\left[1-(1-p)^{n}\right]^{m}} \prod_{i=1}^{m}\left[1-p\left(1-\exp \left\{-\lambda x_{i}\right\}\right)\right]^{n-1}
\end{gathered}
$$

In order to obtain equations of Maximum likelihood estimations (MLE) $\widehat{\lambda}, \widehat{p}$ for parameters $\lambda, p$ we consider

$$
\begin{gathered}
\ln L\left(x_{1}, x_{2}, \ldots, x_{m} ; \lambda, p\right)=m(\ln n+\ln p+\ln \lambda)- \\
\lambda \sum_{i=1}^{m} x_{i}-m \ln \left[1-(1-p)^{n}\right]-(n-1) \sum_{i=1}^{m} \ln \left[1-p\left(1-\exp \left\{-\lambda x_{i}\right\}\right)\right] .
\end{gathered}
$$

Parameter $n$ considered be known, equations of MLE are

$$
\left\{\begin{array}{c}
\frac{\partial \ln L}{\partial \lambda}=\frac{m}{\lambda}-\sum_{i=1}^{m} x_{i}+(n-1) \sum_{i=1}^{m} \frac{p x_{i} \exp \left\{-\lambda x_{i}\right\}}{1-p\left(1-\exp \left\{-\lambda x_{i}\right\}\right)}=0, \\
\frac{\partial \ln L}{\partial p}=\frac{m}{p}-\frac{m n(1-p)^{n-1}}{1-(1-p)^{n}}-(n-1) \sum_{i=1}^{m} \frac{\exp \left\{-\lambda x_{i}\right\}-1}{1-p\left(1-\exp \left\{-\lambda x_{i}\right\}\right)}=0 .
\end{array}\right.
$$

But this equations, with respect to the parameters $\lambda$ and $p$, it is very difficult to solve by means of existing methods. So, EM Algorithm proposed in [1], is more preferable. In this case r.v. $Z$ it is interpreted as missing or latent variable.

Let's consider a sample $\left(\left(x_{1}, z_{1}\right),\left(x_{2}, z_{2}\right), \ldots,\left(x_{m}, z_{m}\right)\right)$ of $n$ observations on the r.v. $(X, Z)$. That means $\left(\left(x_{1}, z_{1}\right),\left(x_{2}, z_{2}\right), \ldots,\left(x_{m}, z_{m}\right)\right)$ may be interpreted as complete data and $\left(x_{1}, x_{2}, \ldots, x_{m}\right)$ as incomplete data's observations.

To formulate EM algorithm we need to know conditional mean value $\mathbb{E}(Z$ $\mid X ; \theta)$, where parameter $\theta=(\lambda, p)$. P.d.f. $u_{\min }(x ; \theta)$ of r.v. $X$, corresponding to the incomplete data, is given by formula (10). But p.d.f. of r.v. $(X, Z)$, corresponding to the complete data, is given by formula

$$
u(x, z ; \theta)=\frac{z \lambda \exp \{-\lambda z x\}}{1-(1-p)^{n}} \mathbb{C}_{n}^{z} p^{z}(1-p)^{n-z} \cdot I_{[0,+\infty)}(x) .
$$


So, p.d.f. of r.v. $Z$ for given (known) $X$, i.e., p.d.f. of r.v. $Z$ conditioned by r.v. $X$, my by expressed, for $x>0$, as

$$
\begin{aligned}
& u(z \mid x ; \theta)=u(x, z ; \theta) / u_{\min }(x ; \theta)= \\
& \frac{\frac{z \lambda \exp \{-\lambda z x\}}{1-(1-p)^{n}} \mathbb{C}_{n}^{k} p^{k}(1-p)^{n-k}}{\frac{n p \lambda \exp \{-\lambda x\}}{1-(1-p)^{n}}[1-p(1-\exp \{-\lambda x\})]^{n-1}}= \\
& \frac{z \lambda \exp \{-\lambda(z-1) x\} \mathbb{C}_{n}^{z} p^{z-1}(1-p)^{n-z}}{n p[1-p(1-\exp \{-\lambda x\})]^{n-1}} .
\end{aligned}
$$

In this way, on the base of $u(z \mid x ; \theta)$, we may calculate conditional mean value

$$
\begin{gathered}
\mathbb{E}(Z \mid X ; \theta) \stackrel{\text { def }}{=} \sum_{z=1}^{n} z \cdot u(z \mid x ; \theta)= \\
\frac{1-p(1-n \exp \{-\lambda x\})}{1-p(1-\exp \{-\lambda x\})} . \quad(\mathbf{1 1})
\end{gathered}
$$

Now, we may describe EM algorithm as iterative approximation of unknown parameter $\theta=(\lambda, p)$ by $\theta^{(h)}=\left(\lambda^{(h)}, p^{(h)}\right)$ calculated at the same step $h \geqslant 1$ such that the condition

$$
\max \left(\left|\lambda^{(h)}-\lambda^{(h-1)}\right|,\left|p^{(h)}-p^{(h-1)}\right|\right) \leqslant \varepsilon \text { or } h=N
$$

was satisfied, where $\varepsilon>0$ is given error and $N$ is pre-established number of iterations.

1. Take $\lambda=\lambda^{(0)}, p=p^{(0)}$ for some $\lambda^{(0)}>0$ and $p^{(0)} \in(0,1)$;

2. Step $\mathbf{E}$ (Expectation): for iteration $h, h \geqslant 1$, calculate expected values $z_{i}^{(h-1)}, i=\overline{1, m}$, according to the formula $(\mathbf{1 1})$

$$
z_{i}^{(h-1)}=\frac{1-p^{(h-1)}\left(1-n \exp \left\{-\lambda^{(h-1)} x_{i}\right\}\right)}{1-p^{(h-1)}\left(1-\exp \left\{-\lambda^{(h-1)} x_{i}\right\}\right)} ;
$$

3. Step M (Maximization): by means of MLE (maximum likelihood estimation) method, taking as sample size $\left(\left(x_{1}, z_{1}^{h-1}\right),\left(x_{2}, z_{2}^{h-1}\right), \ldots,\left(x_{m}, z_{m}^{h-1}\right)\right)$ with likelihood function

$$
\begin{gathered}
L\left(x_{1}, x_{2},, \ldots, x_{m}, z_{1}^{h-1}, z_{2}^{h-1}, \ldots, z_{m}^{h-1} ; \theta^{(h-1)}\right)= \\
\prod_{i=1}^{m} \frac{z_{i}^{h-1} \lambda^{(h-1)} \exp \left\{-\lambda^{(h-1)} z_{i}^{h-1} x\right\}}{1-\left(1-p^{(h-1)}\right)^{n}} \mathbb{C}_{n}^{z_{i}^{h-1}}\left(p^{(h-1)}\right)^{z_{i}^{h-1}}\left(1-p^{(h-1)}\right)^{n-z_{i}^{h-1}},
\end{gathered}
$$


find the next iteration $\theta^{(h)}$ of estimation $\hat{\theta}$ for parameter $\theta=(\lambda, p)$;

4. Check the condition (12). If NOT, then GO TO 2, ELSE $\hat{\theta}:=\theta^{(h)}$, STOP.

Example 1. Let's consider as a statistical data observations, results of $m=10000$ simulations of r.v. $X \sim E B-\operatorname{Min}(\lambda ; n, p)$ and results $\lambda^{(h)}, p^{(h)}$ of estimation of parameters $\lambda, p$ by means of EM algorithm for different values of error $\varepsilon$ and maximal number of iterations $K$.

Case a) $\lambda=4, p=0.25, n=5, \varepsilon=10^{-4}, K=400$ : For initial values $\lambda^{(0)}=\mathbb{E} X, p^{(0)}=\mathbb{D} X$ we have: $h=400, \lambda^{(h)}=4.102892, p^{(h)}=0.22659 ;$

Case b) $\lambda=4, p=0.25, n=2, \varepsilon=10^{-4}, K=500$ : For initial values $\lambda^{(0)}=\mathbb{E} X, p^{(0)}=\mathbb{D} X$ we have: $h=500, \lambda^{(h)}=4.037867, p^{(h)}=0.2724457$.

Example 2. Let's consider the data about life time intervals between two successive strong earthquakes used in [5], see Table 4:

Table 4. Earthquakes in North Anatolia fault zones

$\begin{array}{llll}\text { Date } & \text { Longitude } & \text { Latitude } & \text { Magnitude }(M w) \\ 04.12 .1905 & 39 & 39 & 6.8 \\ 09.02 .1909 & 38 & 40 & 6.3 \\ 25.06 .1910 & 34 & 41 & 6.2 \\ 24.01 .1916 & 36.83 & 40.27 & 7.1 \\ 18.05 .1929 & 37.9 & 40.2 & 6.1 \\ 19.04 .1938 & 33.79 & 39.44 & 6.6 \\ 26.12 .1939 & 39.51 & 39.8 & 7.9 \\ 30.07 .1940 & 35.25 & 39.64 & 6.2 \\ 20.12 .1940 & 39.2 & 39.11 & 6 \\ 08.11 .1941 & 39.5 & 39.74 & 6 \\ 11.12 .1942 & 34.83 & 40.76 & 6.1 \\ 20.12 .1942 & 36.8 & 40.7 & 7 \\ 20.06 .1943 & 30.51 & 40.85 & 6.5 \\ 26.11 .1943 & 33.72 & 41.05 & 7.2 \\ 01.02 .1944 & 32.69 & 41.41 & 7.2 \\ 26.10 .1945 & 33.29 & 41.54 & 6 \\ 13.08 .1951 & 32.87 & 40.88 & 6.9 \\ 07.09 .1953 & 33.01 & 41.09 & 6.4 \\ 20.02 .1956 & 30.49 & 39.89 & 6.4 \\ 26.05 .1957 & 31 & 40.67 & 7.1 \\ 22.07 .1967 & 30.69 & 40.67 & 7.2 \\ 03.09 .1968 & 32.39 & 41.81 & 6.5 \\ 13.03 .1992 & 39.63 & 39.72 & 6.1 \\ 08.03 .1997 & 35.44 & 40.78 & 6 \\ 12.11 .1999 & 31.21 & 40.74 & 7.2\end{array}$


After fitting EB-Min distribution to the above mentioned sample of size $m=25$, using Kolmogorov-Smirnov (K-S) statistics we may compare our results (see the first row of Table 5) with results from [5] (see the last for rows of Table 5).

Table 5. Parameter estimates, Kolmogorov-Smirnov Statistics and p-values from the fit of

\begin{tabular}{|c|c|c|c|}
\hline Distribution & $\begin{array}{l}\text { the each of the } 5 \text { distributio } \\
E M \text { Estimates }\end{array}$ & K-S statistics & $\mathbf{p}-$ values \\
\hline$E B-\operatorname{Min}(\lambda ; 3, p)$ & $\begin{array}{c}\widehat{\theta}=(\widehat{\lambda}, \widehat{p}) \\
=\left(4.68 \times 10^{-4}, 0.585596\right)\end{array}$ & 0.125 & 0.9942 \\
\hline$E P-\operatorname{Min}(\lambda, \mu)$ & $\begin{array}{c}\widehat{\theta}=(\widehat{\lambda}, \widehat{\mu}) \\
=\left(2.6377,3.56 \times 10^{-4}\right)\end{array}$ & 0.0972 & 0.9772 \\
\hline$E G(\lambda, p)$ & $\begin{array}{c}\widehat{\theta}=(\widehat{\lambda}, \widehat{p}) \\
=\left(6.995 \times 10^{-4}, 1.154 \times 10^{-5}\right)\end{array}$ & 0.1839 & 0.3914 \\
\hline$W$ eibull $(\lambda, \mu)$ & $\begin{array}{c}\widehat{\theta}=(\widehat{\lambda}, \widehat{\mu}) \\
=\left(8.12 \times 10^{-4}, 0.7854\right)\end{array}$ & 0.1004 & 0.969 \\
\hline $\operatorname{Gamma}(\lambda, \mu)$ & $\begin{array}{c}\widehat{\theta}=(\widehat{\lambda}, \widehat{\mu}) \\
=\left(4.98 \times 10^{-4}, 0.7117\right)\end{array}$ & 0.1239 & 0.8551 \\
\hline
\end{tabular}

\section{Conclusions}

This paper shows that EB-Min distribution is a good alternative to the EPMin distribution, even to the EG (compounding exponentially distributed lifetimes with zero truncated geometrically distributed r.v.), Weibull or Gamma distributions.

\section{References}

[1] Dempster, A.P., Laird, N.M. and Rubin, D.B.(1977). Maximumlikelihood from incomplete data via the em algorithm, ,J. Royal Statist. Soc. Ser. B. 39: 1-38.

[2] Feller, W. (1965). An introduction to probability theory and its applications. Vol 1, John Wiley\&Sons, New York.

[3] Gonzales, L.A.P., Vaduva, I. (2010). Simulation of some mixed lifetime distributions. The 13-rd Conference of Romanian Society of Probability and Statistics, Technical University of Civil Engineering, Bucharest, April, 16-1\%.

[4] Jose Flores D., Patrick Borges, Vicente G. Cancho, Francisco Louzada, The Complementary exponential power series distribution, Brazilian Journal of Probability and Statistics (to apear) 
[5] Kuş, C. (2007). A new lifetime distribution. Computational StatisticsEDData Analysis 51: 4497-4509.

[6] Leahu, A., Lupu, C.E. (2010). On the binomially mixed exponential lifetime distribution. Proceedings of the Seventh Workshop on Mathematical Modelling of Environmental and Life Sciences Problems, "Ovidius" University, Constanta, September 2008, Ed. Acad. Romana, Bucharest, pp. 191-196.

[7] Lupu Elena Carmen, (2011). Statistical and Mathematical analysis of life data, PhD. Thesis, University of Bucharest, Faculty of Mathematics and Informatics

[8] Vaduva, I. (2005). Models of Simulation. Ed. Univ. Bucharest.

Elena Carmen LUPU,

Department of Pharmacy,

Ovidius University of Constanta,

1, University Alley, Campus, Corp B, Constanta, Romania

Email: clupu@univ-ovidius.ro

Sergiu LUPU,

Department of Nautical Science,

Mircea cel Bătrân" Naval Academy, Constanta,

Fulgerului Street, no.1, 900218, Constanta, Romania.

e-mail: sergiu.lupu@anmb.com

Adina PETCU,

Department of Pharmacy,

Ovidius University of Constanta,

1, University Alley, Campus, Corp B, Constanta, Romania

Email: adinpetcu@yahoo.com 
EB LIFETIME DISTRIBUTIONS AS ALTERNATIVE TO THE EP LIFETIME DISTRIBUTIONS 